\title{
CONSISTENCIA INTERPARENTAL Y SU RELACIÓN CON LA AGRESIÓN Y LA SINTOMATOLOGÍA DEPRESIVA EN NIÑOS Y ADOLESCENTES
}

\author{
Miguel A. Rodríguez, Victoria Del Barrio y Miguel A. Carrasco \\ Facultad de Psicología, Universidad Nacional de Educación a Distancia
}

\begin{abstract}
Resumen: El presente estudio analiza la relación entre la inconsistencia interparental de los hábitos de crianza y la presencia de conductas agresivas y depresivas en una muestra de 1159 sujetos ( $45.3 \%$ varones) de edades comprendidas entre 8 y 17 años. Los resultados muestran que los sujetos procedentes de hogares inconsistentes en control, hostilidad y comunicación, mostraron más conductas agresivas y depresivas que los sujetos de hogares consistentes. La inconsistencia en las estrategias de control interparental fue la variable que en mayor medida predijo las alteraciones estudiadas. No apareció un patrón específico de inconsistencia ligado a la agresión o la sintomatología depresiva del niño, sin embargo, la inconsistencia interparental se asoció especialmente con la primera.
\end{abstract}

Palabras clave: Prácticas de crianza, consistencia o concordancia interparental, ajuste emocional, agresión, depresión.

\section{Interparental consistency and its relationship to aggression and depression in children and adolescents.}

\begin{abstract}
This study analyses the relationship between interparental rearing inconsistency and the presence of aggressive and depressive behaviour in a sample of 1159 subjects (45,3\% male) between 8 and 17 years of age. The results show that subjects from families with rearing inconsistency in control, hostility and communication showed significantly more aggressive and depressive behaviours than subjects from families with interparental consistency. Parents' control inconsistency was the best predictor of the alterations studied. Although no specific pattern of inconsistency associated with child aggression or depressive symptoms was found, interparental inconsistence appeared especially associated with them.
\end{abstract}

Keywords: Rearing, parental consistency, emotional adjustment, aggression, depression.

La forma de educar de los padres es una variable importante en la explicación del ajuste psicosocial de los hijos (Fletcher, Steinberg y Sellers, 1999). Esto ha llevado a algunos auto-

Recibido 18 julio 2008; aceptado 11 noviembre 2008

Correspondencia: Miguel Ángel Carrasco Ortiz, Dpto. Personalidad, Evaluación y Tratamiento Psicológico, Facultad de Psicología, c/ Juan del Rosal 10, 28040 Madrid. Correo-e: macarrasco@psi.uned.es

Esta investigación ha sido subvencionada por el Ministerio de Ciencia y Tecnología dentro de la convocatoria I+D+I, 20012004 mediante el proyecto BSO2001 3042I res a afirmar que el contexto familiar puede suponer para el desarrollo del niño, en unas circunstancias, un factor de riesgo y en otras, de protección a lo largo de la infancia y la adolescencia (Baumrind, 1967, 1971; Collins, Maccoby, Steinberg, Hetherington y Bornstein, 2000; Del Barrio, 1997, 2007; Maccoby y Martin, 1983; Mestre, Pérez y Frías, 1994). Por ello, los valores, orientaciones y comportamientos con los que padres y madres educan a sus hijos parecen tener una importante repercusión sobre la salud psicológica de éstos (Buchanan, Maccoby y Dornbusch, 1996; Lindsey y Caldera, 2005). 
Uno de los componentes de la crianza es la consistencia interparental, es decir, el grado de coincidencia o similitud que padres y madres muestran en las diferentes prácticas educativas (Block, Block y Morrinson, 1981; Lanz, Scabini, Vermulst, y Gerris, 2001; Rodríguez, Del Barrio y Carrasco, 2007; Vaughn, Block y Block, 1988). La concordancia y coherencia entre los hábitos de crianza maternos y paternos contribuye a crear ambientes predecibles y estructurados para los hijos, que facilitan la comprensión de las normas y favorece el cumplimiento de las expectativas que los padres tienen sobre sus hijos (Block et al., 1981; Lindsey y Caldera, 2005). Un contexto de crianza consistente genera seguridad y permite que los hijos establezcan claras relaciones entre su propia conducta y las contingencias asociadas derivadas de la crianza familiar. De esta forma, se genera un adecuado contexto de aprendizaje y desarrollo que facilita la interiorización de las reglas y valores de los padres. El acuerdo entre los padres es un indicador de la paternidad efectiva y de las habilidades parentales para negociar, lo que favorece un estilo educativo basado en la inducción y el apoyo (Deal, Halverson y Wampler, 1989). Alguno de los trabajos más recientes han encontrado que el grado de consistencia entre padres y madres es un predictor tan bueno o mejor del ajuste infantil que variables tales como el desacuerdo entre padres o la calidad marital (Feinberg, Kan y Hetherington, 2007). El desacuerdo o conflicto entre madres y padres facilitan la aparición de estrategias y hábitos de crianza que median en la aparición de problemas infantiles (Schoppe-Sullivan, Schermerhorn, y Cummings, 2007; Sturge-Apple, Davies, Boker, y Cummings, 2004)

Aunque existen numerosos trabajos que han abordado la relación entre la crianza y el desarrollo de manifestaciones emocionales en los hijos (Barber, 1996; Morris, Silk, Steinberg, Sessa, Avenevoli y Essex 2002; Murris, Meesters, Schouten y Hoge, 2004; Pettit y Dodge, 1993; Pianta, Sroufe y Egeland, 1989; Steinberg y Morris, 2001; Sullivan, 2008; Tur, Mestre y Del Barrio, 2004), menos son los estudios que se centran en la consistencia interparental y la presencia de alteraciones emocionales y conductuales en los hijos. Algunas investiga- ciones realizadas sobre este tema se basan mayoritariamente en cuestionarios administrados a madres y padres con hijos de muy corta edad (Block et al., 1981; Deal et al., 1989; Fletcher et al., 1999; Gamble, Ramakumar y Díaz, 2007; Lindsey y Caldera, 2005; Winsler, Madigan y Aquilino, 2005) y con una medida general de inconsistencia. Escasamente, se ha analizado la consistencia interparental, desde la perspectiva de los hijos, en sujetos de más edad y con un análisis específico del patrón de inconsistencia en los diferentes hábitos de crianza. La obtención de información de los hijos sobre la crianza, es de gran relevancia dado el elevado componente de deseabilidad social que muestran los adultos cuando informan sobre su propio comportamiento parental (Roa y Del Barrio, 2001 y 2002) y la baja concordancia entre fuentes informantes (Gaylord, Kitzmann y Coleman, 2003; Paulson y Sputa, 1996; Smetana, 1995).

Entre los hallazgos consultados, la consistencia interparental se ha vinculado con un mejor funcionamiento personal y social. De los estudios que incluyen a sujetos adolescentes, destaca el trabajo de Vaugh et al. (1988) quienes hallaron en una muestra de sujetos entre 3 y 18 años que, según informaban padres y profesores, los hijos procedentes de hogares consistentes obtenían mejores puntuaciones en autocontrol, autoconfianza, control de los impulsos, desarrollo intelectual, comunicación verbal, responsabilidad y orientación hacia la tarea.

Cuando los hogares son inconsistentes, Johnson, Shulman y Collins (1991) encuentran resultados justo en el sentido opuesto: tomando, en este caso, como fuente de información a los propios adolescentes, hallan que aquellos procedentes de hogares poco consistentes, son los adolescentes que manifiestan una menor autoestima, peor adaptación escolar y peores resultados académicos. En la misma línea, Buchanan et al. (1996) encuentran que adolescentes con padres divorciados e inconsistentes tienen mayor número de síntomas de ansiedad, depresión y comportamientos antisociales que aquellos otros adolescentes con padres divorciados pero consistentes. Más recientemente, Feinberg et al. (2007) hallan que la inconsistencia parental predecía la conducta antisocial de los ado- 
lescentes hasta tres años después, aunque no la sintomatología depresiva. Relacionado con la inconsistencia interparental, el conflicto entre padres es también una variable claramente asociada directa o indirectamente con las manifestaciones interiorizadas y exteriorizadas en los adolescentes (ver Schoppe-Sullivan et al., 2007).

Si bien los diferentes trabajos han encontrado que la inconsistencia interparental se asocia con manifestaciones interiorizadas y exteriorizadas en los hijos, no aparecen estudios comparativos que permitan saber específicamente, los hábitos de crianza inconsistentes que pudieran ser responsables de cada una de estas manifestaciones.

El objetivo del presente trabajo es analizar, desde la perspectiva de los hijos, la presencia de agresión y depresión entre sujetos procedentes de hogares inconsistentes frente a los consistentes y explorar el patrón específico de inconsistencia interparental asociado a una manifestación exteriorizada, tal como la agresión, y otra interiorizada, como es la sintomatología depresiva. En este sentido, la obtención de variables exteriorizadas e interiorizadas y su análisis comparativo en relación con la consistencia interparental de diferentes hábitos de crianza puede aportar nuevos resultados sobre la crianza y su repercusión en los hijos.

\section{MÉTODO}

\section{Muestra}

La muestra analizada se compone de un total de 1159 sujetos, 525 varones $(45,3 \%)$ y 634 mujeres $(54,7 \%)$ de edades comprendidas entre 8 y 17 años. La media de edad es 12,17 y su desviación típica 1,61 .

La mayoría de los sujetos proceden de niveles socio-económicos medio y medio-alto (42\%). Pertenecen a familias con 2 hermanos de media, los participantes son primogénitos en el $49,4 \%$ y nacidos en segundo lugar, en el 35,9\%. El $85,5 \%$ de los hijos proceden de hogares en los cuales conviven ambos padres. No existe en el conjunto de la muestra ningún grupo étnico ni cultural diferenciado.

\section{Procedimiento}

En primer lugar, se seleccionaron los sujetos procedentes de diferentes colegios públicos y concertados de la ciudad de Madrid y su área metropolitana. La selección de la muestra se realizó mediante muestreo probabilístico aleatorio, tomando como parámetro poblacional la edad y el sexo. Ambos parámetros fueron estimados a partir del Censo Poblacional de 2001 de la Comunidad de Madrid. Una vez seleccionados, se solicitó la autorización de los centros escolares y de los correspondientes padres. Se excluyeron los sujetos no autorizados y, a posteriori, aquellos cuya madurez lectora era insuficiente. Las pruebas se administraban colectivamente en el grupo clase por psicólogos entrenados y en presencia del profesor. Las presentes medidas formaban parte de una recogida de datos más extensa, inserta en una amplia investigación longitudinal sobre emociones infantiles.

\section{Instrumentos}

Cuestionario de Comportamiento Parental (CRPBI, Schaefer, 1965; adaptado al español por Samper, Cortés, Mestre, Nácher y Tur, 2006; Carrasco, Holgado y Del Barrio, 2007). Este instrumento consta de 52 ítems, cada uno de los cuales se refieren a diferentes hábitos de crianza del padre y de la madre. El autoinforme valora por separado, pero con los mismos elementos, al padre y a la madre. Los factores utilizados fueron los obtenidos mediante análisis factorial exploratorio y confirmatorio por Carrasco et al., (2007). En la presente investigación, sólo se analizaron los siguientes factores de segundo orden: Comunicación, Control y Hostilidad. El primero, evalúa la capacidad de comunicación positiva, implicación, cariño y atención de los padres con el niño (p.ej., «A menudo me alaba»; «Siempre escucha mis ideas y opiniones»); el segundo, evalúa el grado y la forma de control (p.ej., «Quiere controlar todo lo que hago»; «siempre me dice exactamente como debo hacer mi trabajo») y el tercero, la falta de control y atención junto con la actitud de rechazo y negligencia (p.ej., «Actúa como si yo fuera un 
estorbo»; "A menudo dice que soy estúpido»). Todos los ítems fueron evaluados mediante una escala tipo likert con tres niveles de respuesta: 1 «siempre»; 2 «algunas veces»; 3 «nunca». La fiabilidad del instrumento obtenida mediante alfa de Cronbach, fue respectivamente para madres y padres de: 0,86 y 0,87 para Comunicación-afecto; 0,75 y 0,76 para Control autoritario; 0,70 y 0,68 para Hostilidad-negligencia. La fiabilidad test-retest con un año de diferencia fue para madres y padres, respectivamente de: 0,40 y 0,38 para Comunicación; 0,37 y 0,46 para Control; 0,33 y 0,29 para Hostilidad. Diferentes trabajos han documentado adecuadas propiedades psicométricas para este instrumento (Carrasco et al., 2007; Schaefer, 1965; Samper et al., 2006).

Escala Agresividad Física y Verbal (AFV, Caprara y Pastorelli, 1993; adaptada al español por Del Barrio, Moreno y López (2001). La escala de agresividad física y verbal AFV consta de 20 items que evalúan la conducta de hacer daño a otros física y verbalmente. Posee una escala con tres alternativas según la frecuencia de aparición de la conducta (3, «a menudo», 2, «algunas veces» o 1, «nunca»). La escala se compone, además de la puntuación total, de dos subescalas: Agresividad Física (p.ej., «Pego patadas y puñetazos» o «Muerdo a otros») y Agresividad Verbal (p.ej., «Digo tacos»o «Insulto a mis compañeros»). Entre los ítems de la escala, se intercalan diferentes elementos de control, diseñados para controlar la tendencia de respuesta de los sujetos (p.ej., «Veo mucho la televisión»). La fiabilidad y validez mostradas son apropiadas: la consistencia interna evaluadas con el Alpha de Cronbach es de 0.84 y el testretest de 0.77 (Del Barrio, Moreno y López, 2001; Pastorelli, Barbaranelli, Cermak, Rozsa y Caprara, 1997).

Children Depresión Inventory-Short. (CDIS, Kovacs 1992; adaptada al español por Del Barrio, Olmedo y Colodrón, 2002). El cuestionario de depresión infantil abreviado (CDI-S) es una versión de 10 ítem procedente del Inventario de Depresión Infantil de Kovacs (CDI; Kovacs, 1992) en su adaptación al castellano (Del Barrio y Carrasco, 2004). Cada uno de los elementos del test se enuncia mediante tres aseveraciones correspondientes a tres niveles de intensidad de la sintomatología depresiva $(0,1$ y 2). La versión reducida contiene elementos relacionados, entre otras dimensiones, con la baja autoestima (p.ej., «Nadie me quiere»; «Me odio», «Soy feo» o «Me siento solo siempre»), la disforia (p.ej., «Tengo ganas de llorar todos los días», «Estoy triste siempre» 0 «Las cosas me preocupan siempre») o la desesperanza (p.ej., «Nunca me saldrá nada bien», «Todo lo hago mal» o «No tengo amigos»). Del Barrio et al. (2002) encuentran en población normal española un alfa de Cronbach, de 0,71. En la presente muestra el alfa obtenida fue de 0,73.

\section{Análisis estadísticos}

Previamente a la realización de los análisis estadísticos se halló un índice de consistencia interparental en cada uno de los contextos familiares de los sujetos según el siguiente procedimiento: en primer lugar, se halló la varianza entre las puntuaciones alcanzadas por el padre y la madre en cada uno de las variables de crianza estudiadas; en segundo lugar, los valores obtenidos de la varianza se transformaron en puntuaciones tipificadas, para homogeneizar las diferentes escalas, dado el desigual número de ítems en cada una de ellas; finalmente, se realizaron dos grupos de sujetos: aquellos cuyas puntuaciones estuvieron dentro de la media más una desviación típica (entre -1 y 1), los cuales se identificaron como el grupo de sujetos cuyos padres y madres eran consistentes entre sí (consistencia interparen$t a l$ ); y aquellos sujetos cuyas puntuaciones quedaban fuera del rango medio, los cuales se identificaron con el grupo de sujetos de padres y madres inconsistentes entre sí (inconsistencia interparental). De acuerdo con resultados previos (Rodríguez et al., 2007), la consistencia entre padres aparece en más del $90 \%$ de la muestra de estudio en cada uno de los hábitos de crianza analizados.

Obtenidos los diferentes grupos de sujetos pertenecientes a hogares consistentes e inconsistentes, se realizó un análisis de correlaciones entre el conjunto de las variables estudiadas. Las correlaciones entre variables cualitativas dicotómicas se realizaron mediante correlacio- 
nes Rho de Spearman y las correlaciones entre variables cuantitativas mediante correlaciones de Pearson. Junto al análisis de correlaciones se realizaron diferentes análisis unidireccionales de la varianza para obtener la significación de las diferencias entre los grupos consistentes e inconsistentes. En cada uno de estos ANOVAs se halló el tamaño del efecto de las diferencias, mediante el estadístico $d$ de Cohen (1988) y su respectiva correlación $(r)$. Finalmente, un análisis de regresión lineal múltiple con orden jerárquico, tomando como predictores las variables de inconsistencia en cada uno de los hábitos de crianza estudiados y como variables criterio las puntuaciones en agresividad y depresión. Este último análisis, de acuerdo con Cohen, Cohen, West y Aiken (2003), nos permite explorar el porcentaje de varianza explicada de cada uno de los predictores además del incremento en $R^{2}$ que supone la inclusión progresiva de cada una de ellos sobre los que previamente se han incluido. Dado el carácter acumulativo de las variables en cada paso, los predictores incluidos al principio funcionan como variables de control.

\section{RESULTADOS}

\section{Análisis de Correlaciones}

Dado el carácter dicotómico y cualitativo de algunas variables estudiadas (p.ej., consisten- cia interparental y sexo) junto con variables cuantitativas se realizaron correlaciones $R h o$ de Spearman, cuando alguna de las variables era cualitativa y correlaciones de Pearson, cuando éstas eran cuantitativas .Como puede verse en la Tabla 1, tanto las variables de agresión como la depresión, correlacionaron significativamente con las variables de inconsistencia aunque con valores absolutos bajos. Como cabía esperar, las correlaciones entre las puntuaciones de inconsistencia interparental, por una parte, y las relativas a las manifestaciones emocionales, por otra, fueron las más elevadas. Indicando que los padres inconsistentes en una dimensión de la crianza tienden a serlo en el resto de dimensiones. De igual forma, los hijos agresivos, tienden a mostrar también sintomatología depresiva.

Las manifestaciones agresivas correlacionaron más fuertemente con la inconsistencia interparental que las manifestaciones depresivas. Dentro de este grupo de correlaciones, las de mayor valor absoluto aparecieron entre las variables de inconsistencia relativas al control y la hostilidad con las puntuaciones en agresión infantil.

En cuanto a las variables sexo y edad, los varones correlacionaron con la agresión y las mujeres con la depresión. Ser varón se asoció significativamente con la inconsistencia interparental en la comunicación. A mayor edad mayores niveles de agresión y depresión y mayor inconsistencia interparental en la comunicación.

Tabla 1. Análisis de correlaciones para variables cualitativas mediante Rho de Spearman y para variables cuantitativas mediante correlaciones de Pearson

\begin{tabular}{|c|c|c|c|c|c|c|c|c|c|}
\hline & 1 & 2 & 3 & 4 & 5 & 6 & 7 & 8 & 9 \\
\hline 1. Inconsistencia Control & - & & & & & & & & \\
\hline 2. Inconsistencia Hostilidad & $0,29 * *$ & - & & & & & & & \\
\hline 3. Inconsistencia Comunica & $0,22 * *$ & $0,17 * *$ & - & & & & & & \\
\hline 4. Agresividad global & $0,13 * *$ & $0,13^{*}$ & $0,11 * *$ & - & & & & & \\
\hline 5. Agresividad física & $0,12 * *$ & $0,12^{* *}$ & $0,10^{* *}$ & $0,88 * *$ & - & & & & \\
\hline 6. Agresividad verbal & $0,13 * *$ & $0,13 * *$ & $0,11^{*}$ & $0,95 * *$ & 0,74 & - & & & \\
\hline 7. Depresión & $0,06^{*}$ & $0,06^{*}$ & $0,07 * *$ & $0,17 * *$ & $0,15^{* *}$ & $0,16^{* *}$ & - & & \\
\hline 8. Sexo & $-0,02$ & $-0,03$ & $-0,07^{*}$ & $-0,17 * *$ & $-0,20 * *$ & $-0,14 * *$ & $0,08 * *$ & - & \\
\hline 9. Edad & 0,05 & 0,02 & $0,11 * *$ & $0,18^{* *}$ & $0,11^{* *}$ & $0,20 * *$ & $0,18^{* *}$ & 0,05 & - \\
\hline
\end{tabular}

Nota: * $\mathrm{p}<0,05 ; * * \mathrm{p}<0,01$. Sexo (1 «varón»; 2 «mujer»); I = Inconsistencia (1 «inconsistencia», 0 «consistencia») 


\section{Inconsistencia interparental y diferencias en agresión y depresión infantil}

Con el fin de analizar las diferencias entre grupos de sujetos de padres consistentes e inconsistentes se realizaron diferentes análisis univariados de la varianza. Las diferencias encontradas en las manifestaciones de agresión y depresión entre padres consistentes e inconsis- tentes fueron significativas en cada uno de los hábitos de crianza considerados (ver Tabla 2). Los hijos de madres y padres inconsistentes en la comunicación y afecto con los hijos, o inconsistentes en las estrategias de control, e incluso en las expresiones de hostilidad presentaron mayores niveles de sintomatología depresiva así como un mayor número de conductas agresivas tanto física como verbalmente.

Tabla 2. Diferencias entre los hogares consistentes e inconsistentes en cada uno de los factores de crianza y la agresividad y depresión de lo hijos (ANOVA unidireccional)

\begin{tabular}{|c|c|c|c|c|c|c|c|c|c|}
\hline & & $\begin{array}{c}\text { Agresividad } \\
\text { total } \\
M(D T)\end{array}$ & $F_{(1223,1)}$ & $\begin{array}{l}\text { Agresividad } \\
\text { física } \\
M(D T)\end{array}$ & $F_{(1223,1)}$ & $\begin{array}{c}\text { Agresividad } \\
\text { Verbal } \\
M(D T)\end{array}$ & $F_{(1223,1)}$ & $\begin{array}{c}\text { Depresión } \\
M(D T)\end{array}$ & $F_{(1223,1)}$ \\
\hline \multirow{2}{*}{ Comunicación } & 1 & $21,46(4,92)$ & \multirow{2}{*}{$20,31 * *$} & $10,50(2,85)$ & \multirow{2}{*}{$16,99 * *$} & $13,53(3,08)$ & \multirow{2}{*}{$19,42 * *$} & $3,44(2,48)$ & \multirow{2}{*}{$7,498 * *$} \\
\hline & 2 & $24,14(5,92)$ & & $11,93(3,30)$ & & $15,17(3,63)$ & & $4,26(2,91)$ & \\
\hline \multirow{2}{*}{ Control } & 1 & $21,43(4,91)$ & \multirow{2}{*}{$27,20 * *$} & $10,49(2,84)$ & \multirow{2}{*}{$22,98 * *$} & $13,52(3,09)$ & \multirow{2}{*}{$23,66^{* *}$} & $3,42(2,40)$ & \multirow{2}{*}{$16,24 * *$} \\
\hline & 2 & $24,47(5,87)$ & & $12,11(3,50)$ & & $15,29(3,44)$ & & $4,60(3,67)$ & \\
\hline \multirow{2}{*}{ Hostilidad } & 1 & $21,48(4,96)$ & \multirow{2}{*}{$24,87 * *$} & $10,52(2,88)$ & \multirow{2}{*}{$19,28 * *$} & $13,54(3,09)$ & \multirow{2}{*}{$25,88^{* *}$} & $3,45(2,46)$ & \multirow{2}{*}{$8,64 * *$} \\
\hline & 2 & $25,14(5,48)$ & & $12,39(3,27)$ & & $15,87(3,44)$ & & $4,54(3,56)$ & \\
\hline
\end{tabular}

Nota: $* * p<0,01 ; 1$ «padres consistentes»; 2 «padres inconsistentes».

Predicción de la agresividad y la sintomatología depresiva a partir de la inconsistencia interparental

En la Tabla 3, se muestra el análisis de regresión múltiple en orden jerárquico. Se tomaron como predictores las variables de inconsistencia en cada una de las dimensiones de crianza y como variables criterio las manifestaciones de agresividad y depresión. Siguiendo el procedimiento indicado por Cohen et al. (2003), el análisis se realizó en cinco pasos consecutivos, en cada uno de los cuales se incorporó uno de los predictores. Dado el carácter inclusivo de cada uno de los pasos, las variables incorporadas en primer lugar (sexo y edad) funcionaron como variables control. Este procedimiento permite analizar la contribución específica que cada predictor hace a la varianza de la variable dependiente en cada paso y el incremento que supone en la misma respecto de los predictores del paso anterior. Como se desprende de la Tabla 3, cada variable de incon- sistencia supuso un incremento significativo de la varianza explicada, tanto de las manifestaciones exteriorizadas como interiorizadas. Por tanto, la inconsistencia en cada hábito de crianza contribuye significativamente a la aparición de conductas agresivas y depresivas. La inconsistencia en el control interparental fue la variable que más contribuyó a explicar tanto la agresión como la depresión, en segundo lugar, la inconsistencia en la hostilidad y, finalmente, la inconsistencia en la comunicación. La incorporación progresiva de las distintas variables de inconsistencia modificaba los valores de las variables previas ya incluidas lo que indica la relación existente entre los predictores y la posible interacción entre los mismos. El sexo y la edad, consideradas como variables control, fueron las variables con mejor valor predictivo del conjunto de las variables de entrada. Ser varón contribuía a explicar las manifestaciones exteriorizadas y ser mujer las interiorizadas. A mayor edad, mayor presencia de ambas manifestaciones. 
Tabla 3. Análisis de regresión múltiple con orden jerárquico de las variables de inconsistencia interparental sobre las puntuaciones de agresión y depresión en los hijos

\begin{tabular}{|c|c|c|c|c|c|c|c|c|c|c|c|c|}
\hline \multirow[b]{2}{*}{ Predictor } & \multicolumn{3}{|c|}{ Agresión total } & \multicolumn{3}{|c|}{ Agresión física } & \multicolumn{3}{|c|}{ Agresión verbal } & \multicolumn{3}{|c|}{ Depresión } \\
\hline & $\beta$ & $\mathrm{R}_{\text {ajust. }}^{2}$ & $\mathrm{R} \Delta$ & $\beta$ & $\mathrm{R}_{\text {ajust. }}^{2}$ & $\mathrm{R} \Delta$ & $\beta$ & $\mathrm{R}_{\text {ajust. }}^{2}$ & $\mathrm{R} \Delta$ & $\beta$ & $\mathrm{R}_{\text {ajust. }}^{2}$ & $\mathrm{R} \Delta$ \\
\hline Sexo & $0,20 * *$ & 0,041 & $0,041^{*}$ & $0,22 * *$ & 0,052 & $0,052 * *$ & $0,16^{* *}$ & 0,025 & $0,026^{* *}$ & $-0,06^{*}$ & 0,004 & $0,005^{*}$ \\
\hline Sexo & $0,21 * *$ & 0.077 & $0038 *$ & $0,23^{* *}$ & 0068 & 0 & $0,17 * *$ & 0071 & 0 & $-0,05^{*}$ & 0035 & 00 \\
\hline Edad & $0,19^{* *}$ & 0,017 & 0,03 & $0,13^{* *}$ & 0,068 & 0, & $0,21 * *$ & 0 & & $-0,17 * *$ & 0,035 & 0, \\
\hline Sexo & $0,21 * *$ & & & $0,23^{* *}$ & & & $0,17 * *$ & & & $-0,05^{*}$ & & \\
\hline Edad & $0,18^{* *}$ & 0,094 & $0,018^{*}$ & $0,11^{* *}$ & 0,083 & $0,016^{* *}$ & 0,20 ** & 0,086 & $0,016^{* *}$ & 0,16 & 0,043 & $0,009^{* *}$ \\
\hline I. Control & $0,35^{* *}$ & & & $0,12 * *$ & & & $0,12 * *$ & & & 0,09 & & \\
\hline Sexo & $0,20 * *$ & & & $0,23^{* *}$ & & & $0,16^{* *}$ & & & $-0,06^{*}$ & & \\
\hline Edad & $0,17 * *$ & 0100 & $0007 *$ & $0,11 * *$ & 0088 & $0005 *$ & $0,20 * *$ & 0093 & $0007 * *$ & $0,16^{* *}$ & 0045 & 0003 \\
\hline I. Control & $0,11^{* *}$ & & & $0,10^{* *}$ & & & $0,10^{* *}$ & & & $0,07^{*}$ & & \\
\hline I. Hostilidad & $0,08^{* *}$ & & & $0,07 *$ & & & $0,08 * *$ & & & 0,05 & & \\
\hline Sexo & $0,20 * *$ & & & $0,22 * *$ & & & $0,16^{* *}$ & & & $-0,06^{*}$ & & \\
\hline Edad & $0,17 * *$ & & & $0,11 * *$ & & & $0,19 * *$ & & & $0,16^{* *}$ & & \\
\hline I. Control & $0,09^{* *}$ & 0,105 & $0,005^{*}$ & $0,09 * *$ & 0,092 & $0,005^{*}$ & $0,08 * *$ & 0,097 & $0,005^{*}$ & $0,07^{*}$ & 0,046 & 0,001 \\
\hline I. Hostilidad & $0,07^{* *}$ & & & $0,06^{*}$ & & & $0,08 * *$ & & & 0,05 & & \\
\hline I. Comunicac & $0,07 * *$ & & & $0,07 *$ & & & $0,07 *$ & & & 0,03 & & \\
\hline
\end{tabular}

Nota: $* p<0,05 ; * * p<0,01$. Todos los predictores fueron centrados. Sexo (1 «varón»; 0 «mujer»); I = Inconsistencia ( 1 «inconsistencia», 0 «consistencia»). $\beta=$ coeficientes estandarizados

Claramente, la inconsistencia interparental explicaba en mayor medida las manifestaciones exteriorizadas frente a las interiorizadas. No obstante, el patrón de variables predictivas fue similar tanto en la agresividad como en la depresión. El modelo explorado (sexo, edad e inconsistencia interparental) contribuía a explicar la agresión en un $10,5 \%$ y la depresión en un $4,6 \%$.

\section{DISCUSIÓN}

La inconsistencia interparental se relacionó significativamente con las manifestaciones emocionales de los hijos, particularmente, con la agresión y la depresión. Los hijos criados en hogares consistentes interparentalmente fueron los que presentaron menores niveles de sintomatología depresiva y agresión, dando muestras de un mejor ajuste emocional. Como ya encontraron diversos trabajos (Block et al., 1981; Buchanan et al., 1996; Lindsey y Caldera, 2005; Vaugh et al., 1988), la congruencia entre los pa- dres favorece un desarrollo psicológico positivo en los hijos e hijas adolescentes. Los hijos de padres consistentes desarrollan elevados niveles de autocontrol y autoconfianza, los cuales se traducen en un incremento del rendimiento escolar, la responsabilidad y la orientación a la tarea (Block et al., 1981; Vaugh et al., 1988).

Los hijos de padres inconsistentes, frente a los de padres consistentes, en cualquiera de los hábitos de crianza estudiados (control, hostilidad y comunicación) mostraron mayores niveles de depresión y mayor número de conductas agresivas, tanto físicas como verbales. Sin embargo, el análisis detallado de la consistencia interparental en las diferentes dimensiones de crianza, ha puesto de manifiesto que la congruencia en las estrategias de control entre padres y madres es la variable más relevante respecto de la consistencia en la hostilidad, la comunicación o el afecto, y especialmente, en las manifestaciones exteriorizadas.

Trabajos previos consultados son coherentes con estos resultados. La relación entre la in- 
consistencia interparental y la aparición tanto de manifestaciones interiorizadas, tales como la ansiedad y la depresión (Buchanan et al., 1996; Katz y Low, 2004) como exteriorizadas, tales como la agresión (Block et al, 1981; McHale y Rasmussen, 1998; Vaugh et al., 1988), dan apoyo a los presentes resultados. Particularmente, la inconsistencia en el control parental se ha asociado reiteradamente con las manifestaciones exteriorizadas en los hijos (Pettit y Laird, 2002; Pettit, Laird, Dodge, Bates, y Criss, 2001). En el caso de la inconsistencia en el control interparental, los presentes resultados coinciden con la relación directa que Schoppe-Sullivan et al. (2007) hallan entre el conflicto marital y las puntuaciones exteriorizadas de los hijos, relación que no resulta significativa con las puntuaciones interiorizadas de depresión.

Si comparamos, en los resultados hallados en este trabajo, el patrón de consistencia asociado a la agresión, como manifestación emocional exteriorizada, y a la depresión, como manifestación interiorizada, en ambos casos, resultó ser similar. No obstante, el valor predictivo de la consistencia fue destacadamente superior en las manifestaciones exteriorizadas frente a las interiorizadas. Los padres inconsistentes facilitan en mayor medida la aparición de conductas agresivas que depresivas y, en ambas manifestaciones, es la inconsistencia en las estrategias de control la que peor consecuencias educativas genera. La escasez de trabajos que han explorado el patrón específico de inconsistencia en problemas exteriorizados e interiorizados no nos permite hacer comparaciones. Sin embargo, la relevancia de la inconsistencia en el control parental es coherente con aquellos estudios, que establecen una clara relación entre los problemas exteriorizados y la presencia de hábitos de crianza inadecuados relacionados con el control y la gestión de límites: control autoritario, control laxo y permisivo, ausencia de límites o ausencia de supervisión (McFadyen-Ketchum, Bates, Dodge y Pettit, 1996; Pettit y Dodge, 1993). En términos de inconsistencia globalmente considerada, algunos trabajos más recientes, están en sintonía con este patrón diferencial de inconsistencia interparental asociado a las manifestaciones exteriorizadas e interiorizadas. Feinberg (2003) y Feiberg et al. (2007) muestran que el conflicto entre los padres está relacionado con problemas exteriorizados en los hijos pero muy débilmente con la sintomatología depresiva de éstos. El conflicto entre padres, transcurridos tres años, predecía los primeros pero no los segundos (Feiberg et al., 2007). SchoppeSullivan et al. (2007), además de las ya mencionadas relaciones directas entre conflicto parental y conductas exteriorizadas (no interiorizadas) en los hijos, hallan relaciones mediadas entre el conflicto parental y las conductas antisociales por las estrategias de control y de autonomía/apoyo de los padres. Estas relaciones no resultaron significativas con las medidas de depresión de lo hijos. Aunque estos trabajos no evaluaron estrictamente inconsistencia interparental, medidas próximas a ésta como el conflicto o la discordia marital apuntan en la misma dirección que los datos de nuestro trabajo.

Los hogares inconsistentes, de acuerdo con Lindsey y Caldera (2005), son menos predecibles, y en este sentido, son un mejor caldo de cultivo para el desarrollo de manifestaciones agresivas que depresivas. Por tanto, aunque no es posible delimitar un patrón específico de inconsistencia para un tipo u otro de problemas infantiles, si se puede concluir que los hogares inconsistentes funcionan como un factor de riesgo para la aparición de problemas infantiles, especialmente, de los problemas exteriorizados.

Los presentes resultados tienen una importante implicación para la intervención y prevención de los problemas infantiles. Programas dirigidos a la modificación de pautas de crianza inconsistentes, así como la instauración de patrones de crianza compartidos y acordados entre padres y madres han de estar en la base de las escuelas de padres y de la intervención en familias cuyos hijos muestren alteraciones exteriorizadas o interiorizadas.

Finalmente, señalar algunas de las limitaciones presentes en esta investigación, entre las que cabe mencionar: a) un reducido número de sujetos procedentes de hogares inconsistentes, lo cual dificulta la generalización de los resultados; b) la utilización de una sola fuente informante para la obtención de las diferentes medidas pudiera incluir un sesgo en la dirección de los resultados; c) el análisis de los datos des- 
de un diseño transversal, no permite averiguar el alcance de la inconsistencia a lo largo del tiempo; y d) el patrón de inconsistencia evaluado está limitado a los hábitos de crianza procedentes del instrumento utilizado.

Futuros trabajos pudieran subsanar las limitaciones anteriormente mencionadas, y seguir profundizando en el conocimiento de los efectos que la inconsistencia interparental posee sobre el ajuste emocional de los niños y adolescentes.

\section{REFERENCIAS}

Barber, B. (1996). Parental psychological control: revising a neglected construct. Child Development, 67, 32963319.

Baumrind. D. (1967). Child care practices anteceding three patterns of preschool behaviour. Genetic Psychology Monographs, 75, 43-88

Baumrind. D. (1971). Harmonious parents and their preschool children. Developmental Psychology, 4, 99-102.

Block, J., Block, J., y Morrinson, A. (1981). Parental agreement-disagreement on child-rearing orientations and gender-related personality correlates in children. Child Development, 52, 965-974.

Buchanan, C., Maccoby, E., y Dornbusch, S. (1996). Adolescents after divorce. Cambridge, MA: Harvard University Press.

Caprara, G.V., y Pastorelli, C. (1993). Early emotional inestability, prosocial behaviour and aggression: some methodological aspects. European Journal of Personality, 7, 19-36.

Carrasco, M.A., Holgado P., y Del Barrio, V. (2007). Análisis de la estructura del cuestionario de comportamiento parental para niños (CRPBI) en población española. Revista Iberoamericana de Diagnóstico y Evaluación Psicológica 24, 95-120

Cohen, J. (1988). Statistical power analysis for the behavioural sciences $\left(2^{\text {nd }}\right.$ ed.). Hillsdale, NJ: Lawrence Earlbaum Associates

Cohen, J., Cohen, P., West, S.G., y Aiken, L. S. (2003). Applied multiple regression/correlation analysis for the behavioral sciences. Mahwah, NJ: Erlbaum

Collins, W., Maccoby, E., Steinberg, L., Hetherington, M., y Bornstein, M. (2000). Contemporary research on parenting: The case for nature and nurture. American Psychologist, 55, 218-232.

Deal, J. E., Halverson, C. F., y Wampler, K.S. (1989). Parental agreement on child-rearing orientations: Relations to parental, marital, family, and child characteristics. Child Development, 60, 1025-1034.
Del Barrio, V. (1997). Depresión infantil. Barcelona: Ariel. Del Barrio, V. (2007). El niño deprimido. Barcelona: Ariel.

Del Barrio, V., y Carrasco, MA. (2004). Manual del Inventario de depresión infantil CDI. Adaptación española. Madrid: TEA-Ediciones

Del Barrio, V., Moreno, C., y López, R. (2001). Evaluación de la agresión y la inestabilidad emocional en niños españoles: su relación con la depresión. Clínica y $\mathrm{Sa}$ lud, 12, 33-50.

Del Barrio, V., Olmedo, M., y Colodrón D. (2002) Adaptación del CDI-S (Kovacs, 1992) a la población española. Acción Psicológica, 3, 263-272.

Feinberg, M. (2003). The internal structure and ecological context of coparenting: a framework for research and intervention. Parenting: Science and Practice, 3, 95132.

Feinberg, M.E., Kan, M.L., y Hetherington, E.M. (2007). The longitudinal influence of coparenting conflict on parental negativity and adolescent maladjustment. Journal of Marriage and Family, 69, 687-702.

Fletcher, A., Steinberg, L., y Sellers, E. (1999). Adolescents' well-being as a function of perceived interparental consistency. Journal of Marriage and the Family, 61, 599610.

Gamble, W., Ramakumar, S., y Díaz, A. (2007). Maternal and parental similarities and differences in parenting: An examination of Mexican-American parents of young children. Early Childhood Research Quarterly, 22, 7288.

Gaylord, N.K., Kitzmann, M., y Coleman, J.K. (2003). Parent and children's perceptions of parental behaviour: associations with children's psychosocial adjustment in the classroom. Parenting: Science and Practice. 3, 23-47.

Johnson, B.M., Shulman, S., y Collins, W. A. (1991). Systemic patterns of parenting as reported by adolescents: Developmental differences and implications for psychosocial outcomes. Journal of Adolescent Research, 6 , 235-252.

Katz, R., y Low, S.M. (2004). Marital violence, co-parenting, and family level processes in relation to children's adjustment. Journal of Family Psychology, 18, 372-382.

Kovacs, M. (1992). Children's Depression Inventory CDI Manual. New York: Multi-Health Systems.

Lanz, M., Scabini, E., Vermulst, A.A., y Gerris, J.R. (2001). Congruence on child rearing in families with early adolescent and middle adolescent children. International Journal of Behavioral Development, 25, 133-139.

Lindsey, E., y Caldera, Y. (2005). Interparental agreement on the use of control in childrearing and infant's compliance to mother's control strategies. Infant behavior \& Development, 28, 165-178.

Maccoby, E., y Martin, J.A. (1983) Socialization in the context of the family: Parent-child interaction. En P.H. 
Hetherington (Ed.), Handbook of child psychology (vol. 4, pp. 1-102). New York: Wiley.

McFadyen-Ketchum, S., Bates, J., Dodge, K., y Pettit, G. (1996). Patterns of change in early childhood aggressive-disruptive behaviour gender differences in predictions from early coercitive and affectionate mother-child interactions. Child Development, 67, 2417-2433.

McHale, J.P., y Rasmussen, J.L. (1998). Coparental and family group level dynamics during infancy: early family precursors of child and family functioning during preschool. Development \& Psychopathology, 10, 39-59.

Mestre, V., Pérez Delgado, E., y Frías Navarro, D. (1994). Psicopatología familiar: La familia como factor de protección y vulnerabilidad. En E. Pérez Delgado (Coord.), Familia y educación relaciones familiares y desarrollo personal de los hijos (pp. 113-154). Generalitat Valenciana.

Morris, A., Silk, J., Steinberg, L., Sessa, F., Avenevoli, S., y Essex, M. (2002). Temperamental vulnerability and negative parenting as interacting of child adjustment. Journal of Marriage and Family, 64, 461-471.

Muris, P., Meesters, C., Schouten, A., y Hoge, E. (2004). Effects of perceived control on the relationship between perceived parental rearing behaviors and symptoms of anxiety and depression in nonclinical preadolescents. Journal of Youth and Adolescence, 33, 51.

Pastorelli, C., Barbaranelli, C., Cermak, I., Rozsa, S., y Caprara, G.V. (1997). Measuring emotional instability, prosocial behavior and aggression in pre-adolescents: a cross-national study. Personality and Individual Differences, 23, 691-703.

Paulson, E., y Sputa, L. (1996) Patterns of parenting during adolescence: perception of adolescents and parents. Adolescence, 31, 369-381.

Pettit, G., y Dodge, K. (1993). Family interaction patterns and children's conduct problems at home and school: a longitudinal perspective. School Psychology Review, 22, 403-420.

Pettit, G.S., y Laird, R.D. (2002). Psychological control and monitoring in early adolescence: the role of parental involvement and earlier child adjustment. En B.K. Barber (Ed.), Intrusive parenting: how psychological control affects children and adolescents (pp. 97-123). Washington, DC: American Psychological Association.

Pettit, G.S., Laird, R.D., Dodge, K.A., Bates, J.E., y Criss, M. (2001). Antecedents and behaviour-problem outcomes of parental monitoring and psychological control in early adolescence. Child Development, 72, 583-598.
Pianta, R., Sroufe, L., y Egeland, B. (1989). Continuity and discontinuity in maternal sensibility at 6,24 , and 42 months in a high-risk sample. Child Development, 60, 481-487.

Roa, L., y Del Barrio, V. (2001). Adaptación del cuestionario de crianza parental (PCRI-A Gerard, 1994) a la población española. Revista Latinoamericana de Psicología, 33, 329-341.

Roa, L., y Del Barrio, V. (2002). Cuestionario de percepción de crianza para niños y adolescentes. Psicología Educativa, 8, 37-51.

Rodríguez, M.A., Del Barrio V., y Carrasco, M.A. (2007). Consistencia interparental percibida por niños y adolescentes. Revista de Psicología General y Aplicada, 60, 397-410.

Samper, P., Cortés, M., Mestre, V., Nácher, M., y Tur, A. (2006). Adaptación del Child's Report of Parent Behavior Inventory (Schaefer, 1965) a población española. Psicothema, 18, 263-271.

Schaefer, E.S. (1965). Children's reports of parental behaviour: an inventory. Child Development, 36, 413-424.

Schoppe-Sullivan, S., Schermerhorn, A., y Cummings, E. (2007). Marital conflict and children's adjustment: evaluation of the parenting process model. Journal of Marriage and Family, 69, 1118-1134.

Smetana, J. (1995). Parenting styles and conceptions of parental authority during adolescence. Child Development, 66, 299-316.

Steinberg, L., y Morris, A. (2001). Adolescent development. Annual Review of Psychology, 52, 83-110.

Sturge-Apple, M., Davies, P., Boker, S., y Cummings, E.M. (2004). Interparental discord and parenting: testing the moderating roles of child and parent gender. Parenting: Science and Practice, 4, 361-380.

Sullivan, C. (2008). Parenting and internalizing problems: testing models of bidirectional socialization in early childhood. Dissertation Abstracts International: Section B: The Sciences and Engineering, 68 (9-B), 6394.

Tur, A., Mestre, V., y Del Barrio, V. (2004). Factores moduladores de la conducta agresiva y prosocial. El efecto de los hábitos de crianza en la conducta del adolescente. Ansiedad y Estrés, 10, 75-88.

Vaughn, B., Block, J., y Block., J. (1988). Parental agreement on child rearing during early childhood and the psychological characteristics of adolescents. Child Development, 59, 1020-1033.

Winsler, A., Madigan A., y Aquilino, S. (2005). Correspondence between maternal and paternal parenting styles in early childhood. Early Childhood Research Quarterly, 20, 1-12. 